bioRxiv preprint doi: https:/doi.org/10.1101/2022.02.21.481370; this version posted February 22, 2022. The copyright holder for this preprint (which was not certified by peer review) is the author/funder, who has granted bioRxiv a license to display the preprint in perpetuity. It is made available under aCC-BY 4.0 International license.

\title{
LazyPair: scalable prediction of protein-protein interactions and interaction types
}

\author{
Chun Shen Lim, Bikash K. Bhandari ${ }^{1, \dagger}$, Paul P. Gardner ${ }^{1,2, *}$
}

${ }^{1}$ Department of Biochemistry, School of Biomedical Sciences, University of Otago, Dunedin, New Zealand

${ }^{2}$ Biomolecular Interaction Centre, University of Canterbury, Christchurch, New Zealand

${ }^{*}$ Corresponding author. Email: paul.gardner@otago.ac.nz

${ }^{\dagger}$ Current address: European Molecular Biology Laboratory, European Bioinformatics Institute (EMBL-EBI), Wellcome Genome Campus, Hinxton, UK.

\begin{abstract}
Motivation: Almost all cellular processes require protein-protein interactions. Common interaction types include binding, post-translational modifications, and catalysis. However, existing prediction tools do not take these interaction types into account and do not scale well on proteome-wide prediction.

Results: Here we show that a random forest classifier trained on per-residue physicochemical and biochemical properties is useful for predicting protein-protein interactions. Counterintuitively, we find that training random forests by individual interaction types improves accuracy. Furthermore, a combination of these specialised classifiers improves generalisability. We call our protein-protein interaction prediction tool LazyPair. More importantly, LazyPair outperforms the state-of-the-art in accuracy, generalisability and scalability.
\end{abstract}

Availability and implementation

LazyPair and the source code and data for reproducing our analysis are freely available at https://github.com/Gardner-BinfLab/PPI Analysis 2022

and

https://doi.org/10.5281/zenodo.6071630. The web server version and the source code are freely available at https://tisigner.com/lazypair/ and

https://github.com/Gardner-BinfLab/TISIGNER-ReactJS, respectively.

\section{INTRODUCTION}

Protein-protein interactions (PPIs) are involved in nearly all cellular processes and metabolisms. Common experimental methods for detecting PPIs include yeast two-hybrid assays, anti-tag coimmunoprecipitation, and tandem affinity purification. These experimentally validated PPIs are curated and maintained by a number of databases, e.g., BIOGRID (Oughtred et al., 2021), BioPlex (Huttlin et al., 2021), DIP (Salwinski et al., 2004), IntAct (Orchard et al., 2014), mentha (Calderone et al., 2013), MINT (Licata et al., 2012), signor (Licata et al., 2020), and STRING (Szklarczyk et al., 2021). However, these curated PPIs are mostly PPIs detected in human and yeast cells; there is a need to predict PPIs in other species. 
Prediction of PPIs is a non-trivial task. Recent tools (Chen et al., 2019; Hashemifar et al., 2018) have been found to lack generalisability in predicting PPIs across species (Sledzieski et al., 2021). Furthermore, most tools were trained and evaluated using specific studies/databases, raising a question of whether these tools are generalisable across studies/databases. For instance, a tool predominantly trained on PPIs found in yeast two-hybrid assays may be suboptimal in predicting PPIs found in affinity purification-based assays. This is because weak and transient interactions are more likely to be detected in yeast two-hybrid assays than affinity purification-based assays. In addition, existing tools are not scalable for proteome-wide prediction. With these in mind, we aim to develop an accurate, generalisable and scalable PPI prediction tool.

\section{METHODS}

Data

We retrieved PPIs from D-SCRIPT (Sledzieski et al., 2021) and eight databases, namely BIOGRID 4.4.204 (Oughtred et al., 2021), BioPlex 3.0 (Huttlin et al., 2021), DIP 20170205 (Salwinski et al., 2004), IntAct 2021-10-13 (Orchard et al., 2014), mentha 2021-12-20 (Calderone et al., 2013), MINT 2021-12-19 (Licata et al., 2012), signor Jan2022 release (Licata et al., 2020), and STRING 11.5 (Szklarczyk et al., 2021). We selected physical-only PPIs from BIOGRID and STRING.

For specific interaction types, we used the annotations in signor and STRING 10.5 (https://version-10-5.string-db.org/download/protein.actions.v10.5/9606.protein.actions.v10.5.txt. gz).

\section{Machine learning}

We calculated a total of 553 features from protein sequences using the aaindex 1 function of protlearn v0.0.3 (Tadorfer, 2021; Kawashima et al., 2008). AAindex1 is a set of per-residue values that capture different physicochemical and biochemical properties of 20 standard amino acids. Protlearn is a tool to process, calculate and select features from protein sequences. For each protein pair, we took the average value for each feature. We then used these features to train and evaluate random forests using scikit-learn v1.0.2 (Pedregosa et al., 2011).

\section{Benchmarking}

To assess the generalisability of each method, 1000 positive and 1000 negative PPIs were sampled 20 times from each PPI database and low probability PPIs (plnt $<0.1$ in BioPlex 3.0 ), respectively. The redundant PPIs were removed-for some pairs (33.5\%) D-SCRIPT did not produce a result, in order to generate a fair benchmark these were not included in the comparison with LazyPair. AUROC (area under the receiver operating characteristic curve) and AUPRC (area under the precision-recall curve) were computed from 100 bootstrap resamplings of the predicted results, where each sample contained 500 positives and 5000 negatives. In reality, the number of protein pairs that do not interact are likely to be much more frequent than pairs that do, hence our imbalanced positive and negative sets. 
To evaluate scalability, PPI prediction tools were run three times using a single process without GPUs on a high performance computer (Red Hat Enterprise Linux 7.9, 2x 64-core AMD EPYC 7702, 1023GiB memory), and timed using /usr/bin/time -f '\%E' <command>.

\section{Statistical analysis}

Data analysis was done using Pandas v1.2.4 (McKinney, 2010), numpy v1.20.1 (van der Walt et al., 2011), scipy v1.6.2 (Virtanen et al., 2020), and scikit-learn v1.0.2 (Pedregosa et al., 2011). Plots were generated using Matplotlib v3.3.4 (Hunter, 2007), Seaborn v0.11.1 (Waskom et al., 2014), and UpSetPlot v0.6.0 (Nothman, 2021).

\section{Code and data availability}

LazyPair and Jupyter notebooks of our analysis can be found at https://github.com/Gardner-BinfLab/PPI Analysis 2022 and https://doi.org/10.5281/zenodo.6071630. The source code for the LazyPair web application can be found at https://github.com/Gardner-BinfLab/TISIGNER-ReactJS (Bhandari et al., 2021).

\section{RESULTS AND DISCUSSION \\ Physicochemical and biochemical properties of amino acids are useful for predicting PPIs}

We sought to develop an accurate and generalisable PPI prediction tool for proteome-wide analysis. Therefore, we extracted features from protein sequences using AAindex 1 that represents the physicochemical and biochemical properties of amino acids (Kawashima et al., 2008), and trained a random forest for PPI prediction (called Lazy $y_{0}$ ). We used the same datasets that were recently used to develop the state-of-the-art prediction tool D-SCRIPT (Sledzieski et al., 2021). In particular, we used human PPIs for training and PPIs in other model species for evaluation, including Mus musculus, Drosophila melanogaster, Caenorhabditis elegans, Saccharomyces cerevisiae, Escherichia coli. These positive sets were physical-only PPIs originally from STRING 11 (Szklarczyk et al., 2021). The negative sets were protein pairs previously generated by a random sampling approach (Sledzieski et al., 2021; Hashemifar et al., 2018).

The above steps allow us to directly compare the performance results of Lazy ${ }_{0}$ with previously published results for D-SCRIPT (Sledzieski et al., 2021) and PIPR (Chen et al., 2019). In general, Lazy ${ }_{0}$ outperformed PIPR but underperformed D-SCRIPT (Table 1).

Next, we asked how generalisable the prediction tools are across PPIs in different databases. Interestingly, Lazy ${ }_{0}$ outperformed D-SCRIPT in five out of eight instances (Fig 1). However, the performance results presented in Fig 1 that are for PPIs from different databases were approximately $20 \%$ lower for both methods than for the model organism results presented in Table 1. This is likely due to overfitting on human PPIs taken from the STRING database. A closer look revealed that most PPIs were unique across databases (Fig 2). Furthermore, an alternative negative test set was used for Fig 1, i.e., low probability PPIs in BioPlex 3.0 (Huttlin et al., 2021), rather than randomly sampled protein pairs as above. 
Table 1. Performance results for classifiers trained on protein-protein interactions (PPIs) in humans. The performance results for PIPR (Chen et al., 2019) and D-SCRIPT were taken from a previous study (Sledzieski et al., 2021). Both the training and test data sets for the below model organisms were derived from a previous study (Sledzieski et al., 2021).

\begin{tabular}{|c|c|c|c|}
\hline Species & Classifier & AUROC & AUPRC \\
\hline \multirow{3}{*}{$\begin{array}{l}\text { H. sapiens } \\
\text { (5-fold } \\
\text { validation) }\end{array}$} & D-SCRIPT & 0.833 & 0.516 \\
\hline & Lazy $_{0}$ & 0.747 & 0.333 \\
\hline & PIPR & 0.960 & 0.835 \\
\hline \multirow[t]{3}{*}{ M. musculus } & D-SCRIPT & 0.833 & 0.580 \\
\hline & Lazy $_{0}$ & 0.845 & 0.542 \\
\hline & PIPR & 0.839 & 0.526 \\
\hline \multirow[t]{3}{*}{ D. melanogaster } & D-SCRIPT & 0.824 & 0.552 \\
\hline & Lazy $_{0}$ & 0.787 & 0.428 \\
\hline & PIPR & 0.728 & 0.278 \\
\hline \multirow[t]{3}{*}{ C. elegans } & D-SCRIPT & 0.813 & 0.548 \\
\hline & Lazy $_{0}$ & 0.777 & 0.420 \\
\hline & PIPR & 0.757 & 0.346 \\
\hline \multirow[t]{3}{*}{ S. cerevisiae } & D-SCRIPT & 0.789 & 0.405 \\
\hline & Lazy $_{0}$ & 0.755 & 0.348 \\
\hline & PIPR & 0.718 & 0.230 \\
\hline \multirow[t]{3}{*}{ E. coli } & D-SCRIPT & 0.863 & 0.571 \\
\hline & Lazy $_{0}$ & 0.787 & 0.464 \\
\hline & PIPR & 0.675 & 0.308 \\
\hline
\end{tabular}

Boldface indicates the highest scores. AUROC, area under the receiver operating characteristic curve; AUPRC, area under the precision-recall curve. 


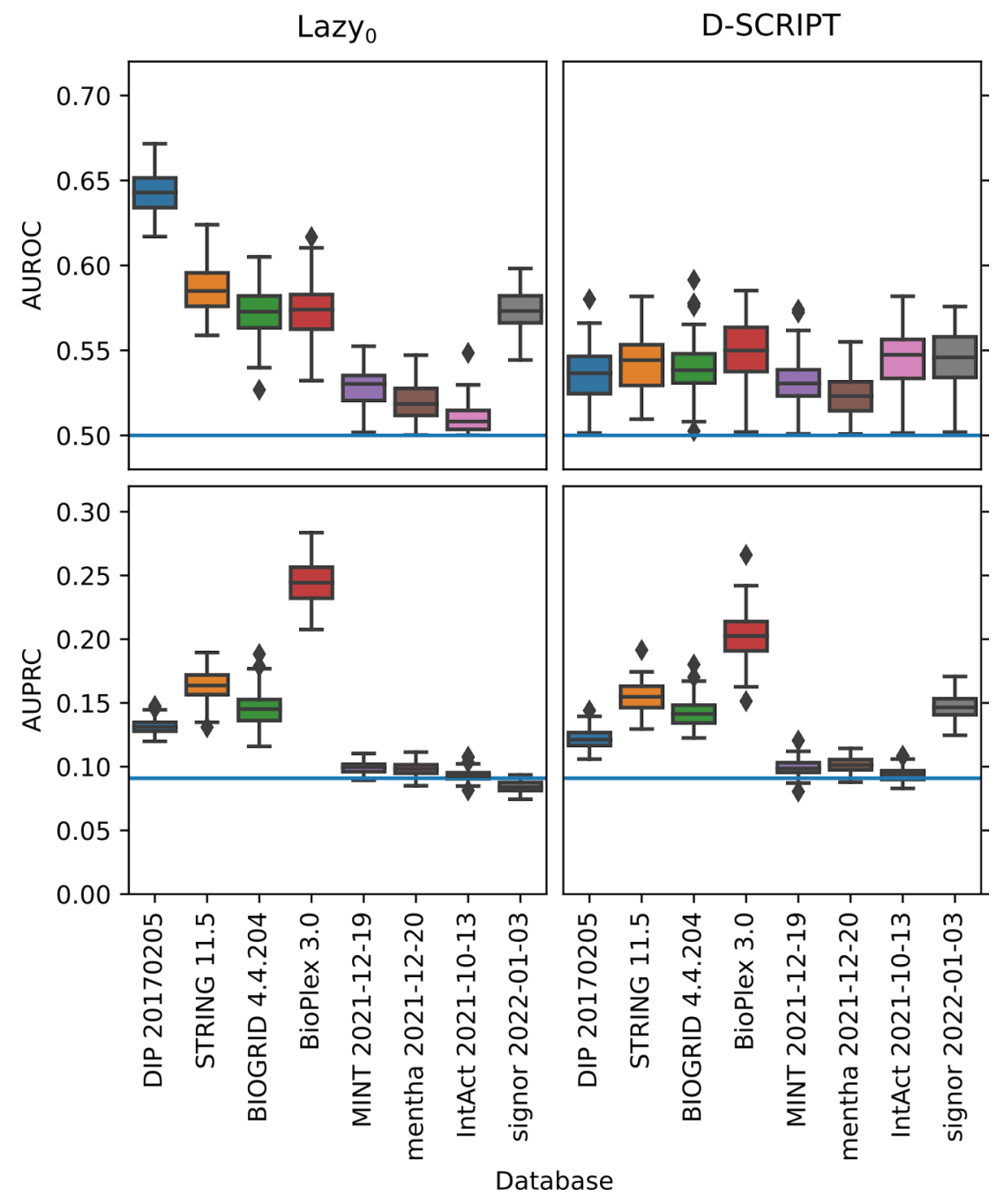

Fig 1. Random forest (Lazy $)_{0}$ trained on physical protein-protein interactions (PPIs) in humans is more generalisable across PPI databases than that of convolutional neural networks. Low probability PPIs were used as the negative test set (plnt <0.1 in BioPlex 3.0). The performance results of Lazy ${ }_{0}$ and D-SCRIPT were computed from 100 bootstrap replicates. The blue lines indicate the performance results of random classifiers. AUPRC, area under the precision-recall curve; AUROC, area under the receiver operating characteristic curve. 


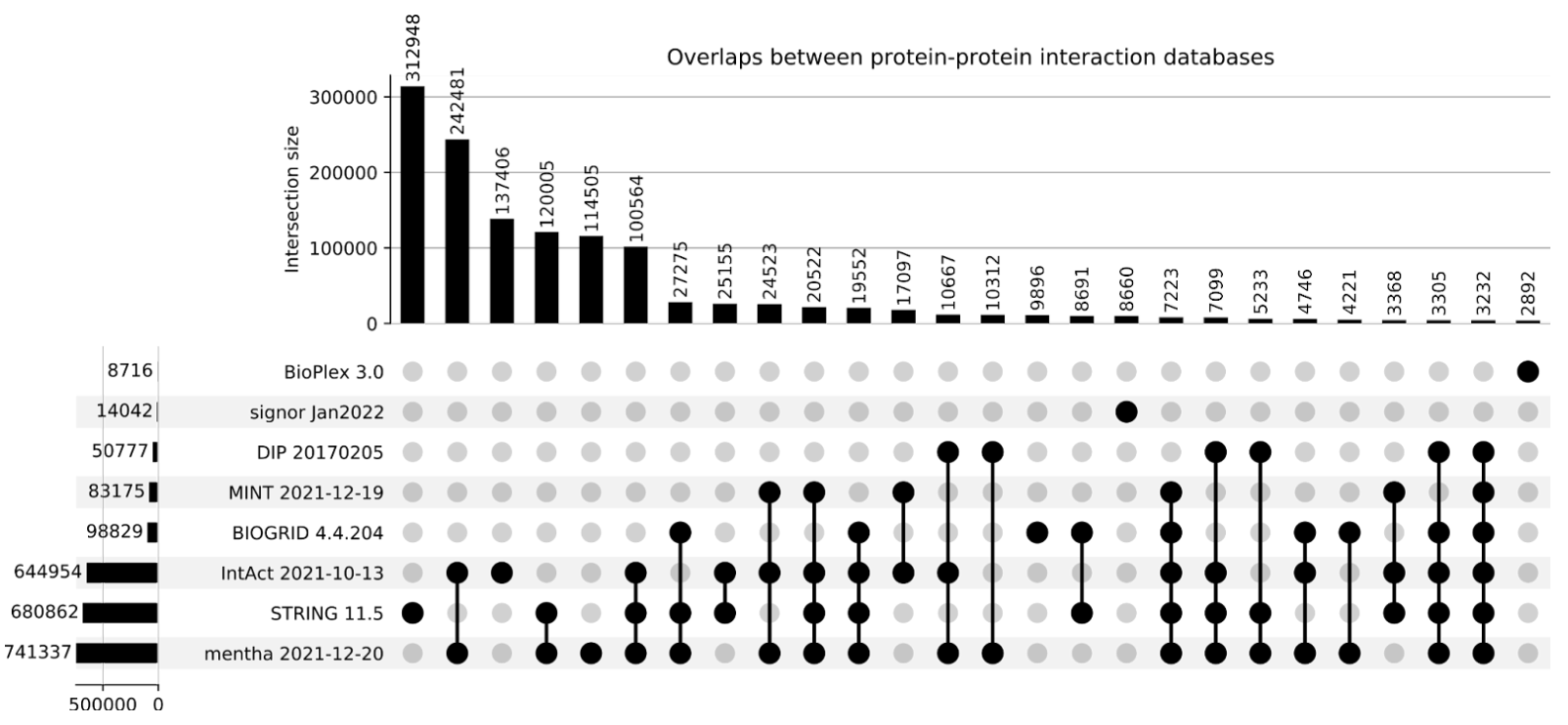

Fig 2. Most PPIs are unique across databases. Physical-only PPI releases were used whenever possible, as for BIOGRID and STRING.

\section{Random forests trained by specific interaction types show improved accuracy and generalisability}

We reasoned that the generalisability issue may be amplified by the complexity of protein-protein interaction types. As some interaction types are more transient than others, e.g. phosphorylation versus binding/assembly, transient and stable PPIs may be attributed to distinct sequence properties. Therefore, we divided the PPIs in STRING and signor by interaction types and separately trained random forests on these subsets. We did not use the interaction types in other databases as only high-level interaction types were available, i.e., association (mi:0914), physical association (Ml:0915), and direct interaction (Ml:0407).

Interestingly, most of the specialised classifiers outperformed the generic classifier (Fig 3 versus Fig 1, Lazy ${ }_{1-10}$ versus Lazy $)_{0}$. We ranked the features by Gini importance and found that a "hydrophobicity coefficient" (WILM950103) is among the strongest features for these classifiers. There is a strong correlation between feature strengths for "binding", "catalysis" and "reaction" interaction types-the sequence features most strongly associated with these are hydrophobicity, alpha and turn propensities, and an "other" class of AAindex1 (Supplementary Figs S1). However, many of these specialised classifiers also have distinct feature rankings, suggesting that proteins involved in different interaction types are distinct (Supplementary Figs S1 and S2, Spearman's correlation coefficients $\sim 0.5$ ).

We also found that the median probability of the specialised classifiers improved generalisability (Fig 4). We then repeated the training step using the complete datasets/subsets and combined these classifiers as a PPI prediction tool called LazyPair. 

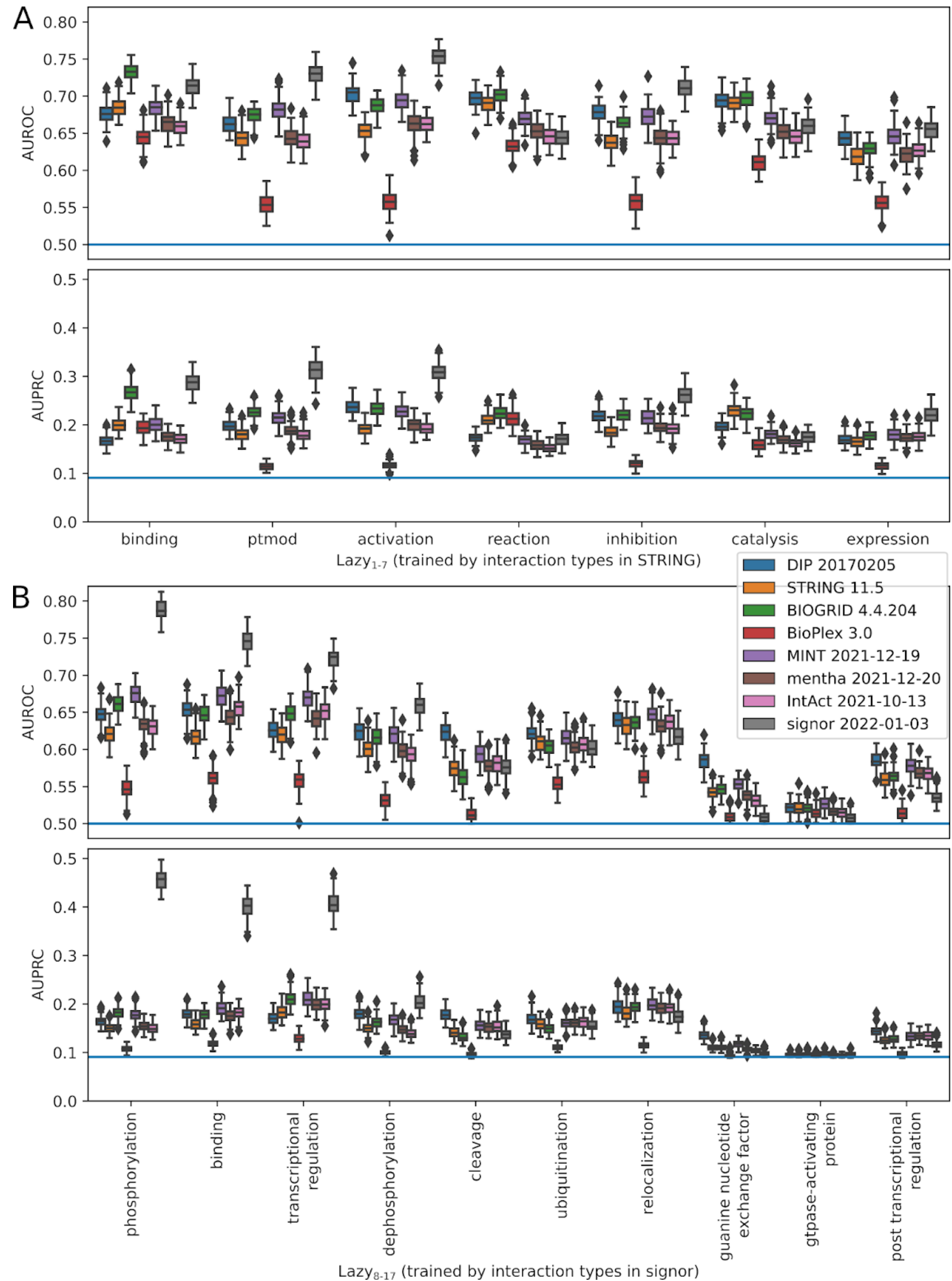
Fig 3. Random forests $\left(\operatorname{Lazy}_{1-17}\right)$ trained by specific interaction types show improved performance results. (A) All 7 interaction types in STRING 10.5 were used for training, including binding $(n=15,925)$, ptmod (post-translational modification, $n=926)$, activation $(n=1,283)$, reaction $(n=7,295)$, inhibition $(n=542)$, catalysis $(n=3,525)$, expression $(n=265)$. (B) The 10 largest interaction types in signor were used for training, including phosphorylation $(n=2,554)$, binding $(n=2,153)$, transcriptional regulation $(n=2,484)$, dephosphorylation $(n=254)$, cleavage $(n=156)$, ubiquitination $(n=65)$, relocalization $(n=138)$, guanine nucleotide exchange factor $(n=27)$, gtpase-activating protein $(n=29)$, post transcriptional regulation $(n=51)$. For negative PPIs, two independent sets of proteins with low probability PPIs were used for training and evaluation (plnt $<0.1$ in BioPlex 3.0). The performance results of Lazy ${ }_{1-17}$ were computed from 100 bootstrap resampling. The blue lines indicate the performance results of random classifiers. AUPRC, area under the precision-recall curve; AUROC, area under the receiver operating characteristic curve.

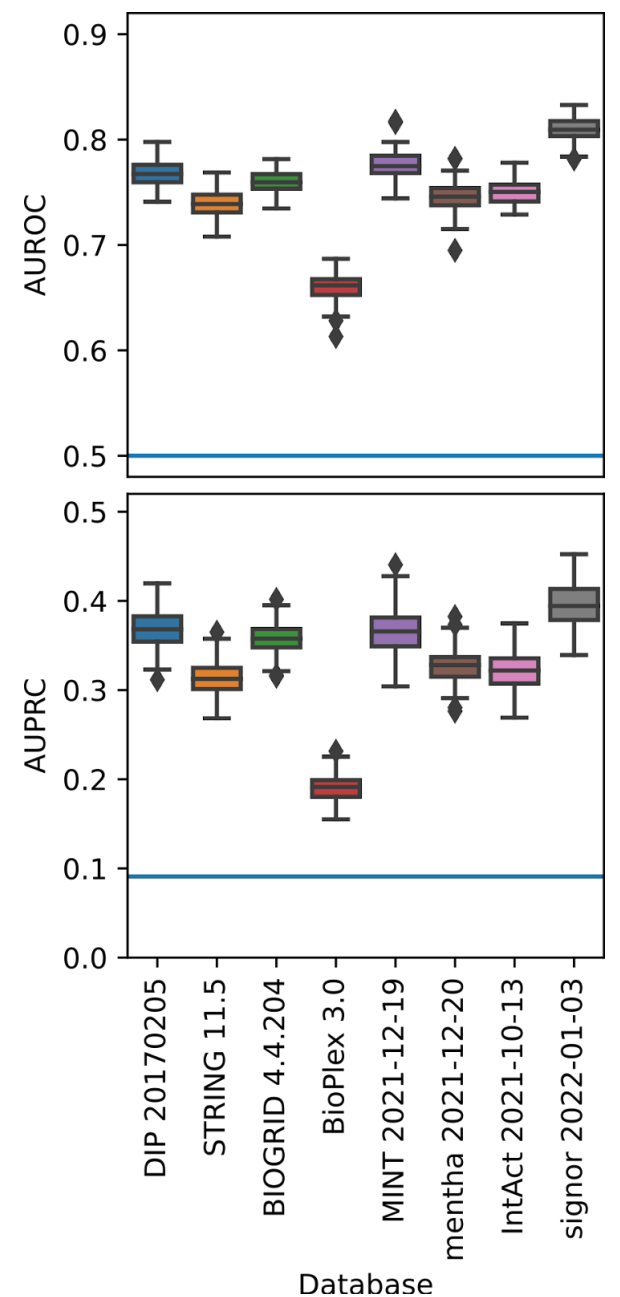

Fig 4. median probabilities of $\operatorname{Lazy}_{0-17}$ improve generalisability. The performance results were computed from 100 bootstrap resampling. The blue lines indicate the performance results of random classifiers. AUPRC, area under the precision-recall curve; AUROC, area under the receiver operating characteristic curve. 


\section{LazyPair is scalable for proteome-wide PPI prediction}

For scalability benchmarking, we tested the effects of (i) protein sequence length and (ii) sample size. LazyPair outperformed the state-of-the-art prediction tool in both tests (Fig 4).

In the first test, we first selected single sequences spanning across the distribution of sequence lengths from PPI databases (Fig 4A). We then retrieved the interacting partners and tested the PPI prediction tools three times (Fig 4B). LazyPair took approximately $O(n)$ with negligible wall time as the features were simply the arithmetic means of per-residue values (AAindex1).

In the second test, we performed random sampling by increasing the sample size of PPI test sets. This allowed us to obtain the test sets in ascending order of magnitude and further test the PPI prediction tools three times (Fig 4B). LazyPair only showed signs of slowing down at 1,000 protein pairs $(12.2 \pm 1.0 \mathrm{~s}$ versus $2 \mathrm{~h} 44 \mathrm{~min} 10.7 \pm 9 \mathrm{~min} 3.4 \mathrm{~s}$, using a single process without GPUs; 2×64-core AMD EPYC 7702, 1023GiB memory).

To attain proteome-wide PPI prediction, we have implemented multiprocessing in LazyPair. We found that LazyPair took $2 \mathrm{~h} 17 \mathrm{~min} 39.7 \mathrm{~s} \pm 8 \mathrm{~min} 21.4 \mathrm{~s}$ to predict all 9,644,832 possible PPIs in E. coli (UniProt: UP000000625; 4392 proteins) using 128 parallel processes.

In conclusion, we have developed an accurate and generalisable PPI prediction tool that is capable of predicting proteome-wide PPIs. To make LazyPair accessible to a broader audience, we have also developed a web version available at https://tisigner.com/lazypair.
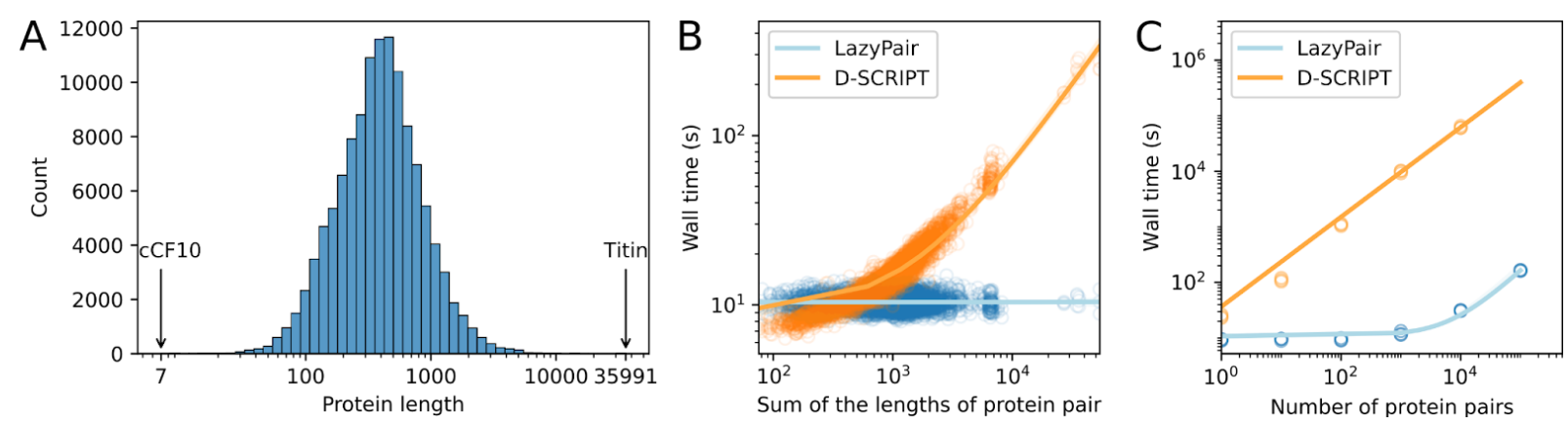

Fig 4. LazyPair is scalable for large-scale PPI prediction. (A) Distribution of the lengths of protein sequences from PPI databases. The shortest sequence is the 7-residue bacterial sex pheromone CCF10, whereas the longest sequence is the 35,991-residue human titin. (B) Wall time of PPI prediction tools for single protein pairs. LazyPair took approximately $O(n)$ time with increase in length of the interacting pairs. (C) Wall time of PPI prediction tools for different sample sizes. 


\section{ACKNOWLEDGEMENTS}

This work was supported by the Royal Society of New Zealand Te Apārangi [Marsden grant: 19-UOO-040 to P.P.G.] and the Ministry of Business, Innovation and Employment [MBIE Data Science Programmes grant: UOAX1932 to P.P.G.].

\section{AUTHOR CONTRIBUTIONS}

C.S.L. and P.P.G. conceived the study; C.S.L. analysed the data and developed LazyPair; B.K.B. developed the LazyPair web application; C.S.L. drafted the manuscript; C.S.L., B.K.B., and P.P.G. reviewed, edited, and approved the manuscript.

\section{COMPETING INTERESTS}

The authors declare no competing interests.

\section{REFERENCES}

Bastolla,U. et al. (2005) Principal eigenvector of contact matrices and hydrophobicity profiles in proteins. Proteins, 58, 22-30.

Bhandari,B.K. et al. (2021) TISIGNER.com: web services for improving recombinant protein production. Nucleic Acids Res., 49, W654-W661.

Calderone,A. et al. (2013) mentha: a resource for browsing integrated protein-interaction networks. Nat. Methods, 10, 690-691.

Chen,M. et al. (2019) Multifaceted protein-protein interaction prediction based on Siamese residual RCNN. Bioinformatics, 35, i305-i314.

Hashemifar,S. et al. (2018) Predicting protein-protein interactions through sequence-based deep learning. Bioinformatics, 34, i802-i810.

Hunter,J.D. (2007) Matplotlib: A 2D Graphics Environment. Comput. Sci. Eng., 9, 90-95.

Huttlin,E.L. et al. (2021) Dual proteome-scale networks reveal cell-specific remodeling of the human interactome. Cell, 184, 3022-3040.e28.

Kawashima,S. et al. (2008) AAindex: amino acid index database, progress report 2008. Nucleic Acids Res., 36, D202-5.

Licata,L. et al. (2012) MINT, the molecular interaction database: 2012 update. Nucleic Acids Res., 40, D857-61.

Licata,L. et al. (2020) SIGNOR 2.0, the SIGnaling Network Open Resource 2.0: 2019 update. Nucleic Acids Res., 48, D504-D510.

McKinney,W. (2010) Data Structures for Statistical Computing in Python. In, Proceedings of the 9th Python in Science Conference., pp. 51-56.

Miyazawa,S. and Jernigan,R.L. (1999) Self-consistent estimation of inter-residue protein contact energies based on an equilibrium mixture approximation of residues. Proteins, 34, 49-68.

Nishikawa,K. and Ooi,T. (1986) Radial locations of amino acid residues in a globular protein: correlation with the sequence. J. Biochem., 100, 1043-1047.

Nothman,J. (2021) UpSetPlot: Draw UpSet plots with Matplotlib.

Orchard,S. et al. (2014) The MIntAct project--IntAct as a common curation platform for 11 molecular interaction databases. Nucleic Acids Res., 42, D358-63.

Oughtred,R. et al. (2021) The BioGRID database: A comprehensive biomedical resource of curated protein, genetic, and chemical interactions. Protein Sci., 30, 187-200. 
bioRxiv preprint doi: https://doi org/10.1101/2022 $0221.481370 \cdot$ this version posted February 22,2022 . The copyright holder for this

Pedregosa,F. et al. (2011) Scikit-learn: Machine Learning in Python. J. Mach. Learn. Res., 12, 2825-2830.

Salwinski, L. et al. (2004) The Database of Interacting Proteins: 2004 update. Nucleic Acids Res., 32, D449-51.

Sledzieski,S. et al. (2021) D-SCRIPT translates genome to phenome with sequence-based, structure-aware, genome-scale predictions of protein-protein interactions. Cell Syst, 12, 969-982.e6.

Szklarczyk,D. et al. (2021) The STRING database in 2021: customizable protein-protein networks, and functional characterization of user-uploaded gene/measurement sets. Nucleic Acids Res., 49, D605-D612.

Tadorfer,T. (2021) protlearn: A Python package for extracting protein sequence features.

Virtanen,P. et al. (2020) SciPy 1.0: fundamental algorithms for scientific computing in Python. Nat. Methods, 17, 261-272.

van der Walt,S. et al. (2011) The NumPy Array: A Structure for Efficient Numerical Computation. Comput. Sci. Eng., 13, 22-30.

Waskom,M. et al. (2014) seaborn: v0.5.0 (November 2014). 